\title{
A PROOF OF KAMP'S THEOREM
}

\author{
ALEXANDER RABINOVICH
}

The Blavatnik School of Computer Science, Tel Aviv University e-mail address: rabinoa@post.tau.ac.il

ABStract. We provide a simple proof of Kamp's theorem.

\section{INTRODUCTION}

Temporal Logic $(T L)$ introduced to Computer Science by Pnueli in 10] is a convenient framework for reasoning about "reactive" systems. This has made temporal logics a popular subject in the Computer Science community, enjoying extensive research in the past 30 years. In $T L$ we describe basic system properties by atomic propositions that hold at some points in time, but not at others. More complex properties are expressed by formulas built from the atoms using Boolean connectives and Modalities (temporal connectives): A $k$-place modality $M$ transforms statements $\varphi_{1}, \ldots, \varphi_{k}$ possibly on 'past' or 'future' points to a statement $M\left(\varphi_{1}, \ldots, \varphi_{k}\right)$ on the 'present' point $t_{0}$. The rule to determine the truth of a statement $M\left(\varphi_{1}, \ldots, \varphi_{k}\right)$ at $t_{0}$ is called a truth table of $M$. The choice of particular modalities with their truth tables yields different temporal logics. A temporal logic with modalities $M_{1}, \ldots, M_{k}$ is denoted by $T L\left(M_{1}, \ldots, M_{k}\right)$.

The simplest example is the one place modality $\diamond P$ saying: " $P$ holds some time in the future." Its truth table is formalized by $\varphi_{\diamond}\left(x_{0}, P\right):=\exists x\left(x>x_{0} \wedge P(x)\right)$. This is a formula of the First-Order Monadic Logic of Order (FOMLO) - a fundamental formalism in Mathematical Logic where formulas are built using atomic propositions $P(x)$, atomic relations between elements $x_{1}=x_{2}, x_{1}<x_{2}$, Boolean connectives and first-order quantifiers $\exists x$ and $\forall x$. Two more natural modalities are the modalities Until ("Until") and Since ("Since"). $X$ Until $Y$ means that $X$ will hold from now until a time in the future when $Y$ will hold. $X$ Since $Y$ means that $Y$ was true at some point of time in the past and since that point $X$ was true until (not necessarily including) now. Both modalities have truth tables in FOMLO. Most modalities used in the literature are defined by such FOMLO truth tables, and as a result, every temporal formula translates directly into an equivalent FOMLO formula. Thus, the different temporal logics may be considered as a convenient way to use fragments of FOMLO. FOMLO can also serve as a yardstick by which one is able to check the strength of temporal logics: A temporal logic is expressively complete for

2012 ACM CCS: [Theory of computation]: Logic-Modal and temporal logics.

Key words and phrases: Temporal Logic, Monadic Logic, Expressive Completeness. 
a fragment $L$ of FOMLO if every formula of $L$ with a single free variable $x_{0}$ is equivalent to a temporal formula.

Actually, the notion of expressive completeness refers to a temporal logic and to a model (or a class of models), since the question whether two formulas are equivalent depends on the domain over which they are evaluated. Any (partially) ordered set with monadic predicates is a model for $T L$ and $F O M L O$, but the main, canonical, linear time intended models are the non-negative integers $\langle\mathbb{N},<\rangle$ for discrete time and the reals $\langle\mathbb{R},<\rangle$ for continuous time.

Kamp's theorem [8] states that the temporal logic with modalities Until and Since is expressively complete for FOMLO over the above two linear time canonica 1 models.

This seminal theorem initiated the whole study of expressive completeness, and it remains one of the most interesting and distinctive results in temporal logic; very few, if any, similar 'modal' results exist. Several alternative proofs of it and stronger results have appeared; none of them are trivial (at least to most people) [7].

The objective of this paper is to provide a simple proof of Kamp's theorem.

The rest of the paper is organized as follows: In Section 2 we recall the definitions of the monadic logic, the temporal logics and state Kamp's theorem. Section 3 introduces formulas in a normal form and states their simple properties. In Section 4 we prove Kamp's theorem. The proof of one proposition is postponed to Section 5. Section 6 comments on the previous proofs of Kamp's theorem. Finally, in Section 7, we show that our proof can be easily modified to prove expressive completeness for the future fragment of FOMLO.

\section{Preliminaries}

In this section we recall the definitions of the first-order monadic logic of order, the temporal logics and state Kamp's theorem.

Fix a set $\Sigma$ of atoms. We use $P, Q, R, S \ldots$ to denote members of $\Sigma$. The syntax and semantics of both logics are defined below with respect to such $\Sigma$.

2.1. First-Order Monadic Logic of Order. Syntax: In the context of FOMLO, the atoms of $\Sigma$ are referred to (and used) as unary predicate symbols. Formulas are built using these symbols, plus two binary relation symbols: $<$ and $=$, and a set of first-order variables (denoted: $x, y, z, \ldots$ ). Formulas are defined by the grammar:

$$
\begin{gathered}
\text { atomic }::=x<y|x=y| P(x) \text { (where } P \in \Sigma \text { ) } \\
\varphi::=\text { atomic }\left|\neg \varphi_{1}\right| \varphi_{1} \vee \varphi_{2}\left|\varphi_{1} \wedge \varphi_{2}\right| \exists x \varphi_{1} \mid \forall x \varphi_{1}
\end{gathered}
$$

We also use the standard abbreviated notation for bounded quantifiers, e.g., $(\exists x)_{>z}(\ldots)$ denotes $\exists x((x>z) \wedge(\ldots))$, and $(\forall x)^{<z}(\ldots)$ denotes $\forall x((x<z) \rightarrow(\ldots))$, and $\left((\forall x)_{>z_{1}}^{<z_{2}}(\ldots)\right.$ denotes $\forall x\left(\left(z_{1}<x<z_{2}\right) \rightarrow(\ldots)\right)$, etc.

Semantics. Formulas are interpreted over labeled linear orders which are called chains. A $\Sigma$-chain is a triplet $\mathcal{M}=(T,<, \mathcal{I})$ where $T$ is a set - the domain of the chain, $<$ is a linear order relation on $T$, and $\mathcal{I}: \Sigma \rightarrow \mathcal{P}(T)$ is the interpretation of $\Sigma$ (where $\mathcal{P}$ is the powerset notation). We use the standard notation $\mathcal{M}, t_{1}, t_{2}, \ldots t_{n} \models \varphi\left(x_{1}, x_{2}, \ldots x_{n}\right)$ to indicate that the formula $\varphi$ with free variables among $x_{1}, \ldots, x_{n}$ is satisfiable in $\mathcal{M}$ when

\footnotetext{
$1_{\text {the technical notion which unifies }}\langle\mathbb{N},<\rangle$ and $\langle\mathbb{R},<\rangle$ is Dedekind completeness.
} 
$x_{i}$ are interpreted as elements $t_{i}$ of $\mathcal{M}$. For atomic $P(x)$ this is defined by: $\mathcal{M}, t \models P(x)$ iff $t \in \mathcal{I}(P)$; the semantics of $<,=, \neg, \wedge, \vee, \exists$ and $\forall$ is defined in a standard way.

2.2. $T L$ (Until, Since) Temporal Logic. In this section we recall the syntax and semantics of a temporal logic with strict-Until and strict-Since modalities, denoted by TL(Until, Since).

In the context of temporal logics, the atoms of $\Sigma$ are used as atomic propositions (also called propositional atoms). Formulas of $T L$ (Until, Since) are built using these atoms, Boolean connectives and strict-Until and strict-Since binary modalities. The formulas are defined by the grammar:

$$
F::=\text { True }|P| \neg F_{1}\left|F_{1} \vee F_{2}\right| F_{1} \wedge F_{2} \mid F_{1} \text { Until } F_{2} \mid F_{1} \text { Since } F_{2},
$$

where $P \in \Sigma$.

Semantics. Formulas are interpreted at time-points (or moments) in chains (elements of the domain). The semantics of $T L$ (Until, Since) formulas is defined inductively: Given a chain $\mathcal{M}=(T,<, \mathcal{I})$ and $t \in T$, define when a formula $F$ holds in $\mathcal{M}$ at $t$-denoted $\mathcal{M}, t \models F$ :

- $\mathcal{M}, t \models P$ iff $t \in \mathcal{I}(P)$, for any propositional atom $P$.

- $\mathcal{M}, t \models F_{1} \vee F_{2}$ iff $\mathcal{M}, t \models F_{1}$ or $\mathcal{M}, t \models F_{2}$; similarly for $\wedge$ and $\neg$.

- $\mathcal{M}, t \models F_{1}$ Until $F_{2}$ iff there is $t^{\prime}>t$ such that $\mathcal{M}, t^{\prime} \models F_{2}$ and $\mathcal{M}, t_{1} \models F_{1}$ for all $t_{1} \in\left(t, t^{\prime}\right)$.

- $\mathcal{M}, t \models F_{1}$ Since $F_{2}$ iff there is $t^{\prime}<t$ such that $\mathcal{M}, t^{\prime} \models F_{2}$ and $\mathcal{M}, t_{1} \models F_{1}$ for all $t_{1} \in\left(t^{\prime}, t\right)$.

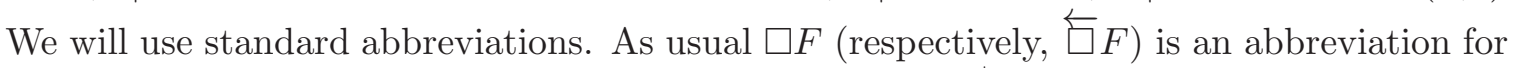
$\neg\left(\right.$ TrueUntil $(\neg F)$ ) (respectively, $\neg($ TrueSince $(\neg F))$ ), and $\mathbf{K}^{+}(F)$ (respectively, $\mathbf{K}^{-}(F)$ ) is an abbreviation for $\neg((\neg F)$ UntilTrue) (respectively, $\neg((\neg F)$ SinceTrue $))$.

(1) $\square F$ (respectively, $\overleftarrow{\square} F$ ) holds at $t$ iff $F$ holds everywhere after (respectively, before) $t$.

(2) $\mathbf{K}^{-}(F)$ holds at a moment $t$ iff $t=\sup \left(\left\{t^{\prime} \mid t^{\prime}<t\right.\right.$ and $F$ holds at $\left.\left.t^{\prime}\right\}\right)$.

(3) $\mathbf{K}^{+}(F)$ holds at a moment $t$ iff $t=\inf \left(\left\{t^{\prime} \mid t^{\prime}>t\right.\right.$ and $F$ holds at $\left.\left.t^{\prime}\right\}\right)$.

Note that $\mathbf{K}^{+}$(True) (respectively, $\mathbf{K}^{-}$(True)) holds at $t$ in $\mathcal{M}$ if $t$ is a right limit (respectively, a left limit) point of the underlining order. In particular, both $\mathbf{K}^{+}$(True) and $\mathbf{K}^{-}$(True) are equivalent to False in the chains over $(\mathbb{N},<)$,

2.3. Kamp's Theorem. Equivalence between temporal and monadic formulas is naturally defined: $F$ is equivalent to $\varphi(x)$ over a class $\mathcal{C}$ of structures iff for any $\mathcal{M} \in \mathcal{C}$ and $t \in \mathcal{M}$ : $\mathcal{M}, t \models F \Leftrightarrow \mathcal{M}, t \models \varphi(x)$. If $\mathcal{C}$ is the class of all chains, we will say that $F$ is equivalent to $\varphi$.

A linear order $(T,<)$ is Dedekind complete if for every non-empty subset $S$ of $T$, if $S$ has a lower bound in $T$ then it has a greatest lower bound, written $\inf (S)$, and if $S$ has an upper bound in $T$ then it has a least upper bound, written $\sup (S)$. The canonical linear time models $(\mathbb{N},<)$ and $(\mathbb{R},<)$ are Dedekind complete, while the order of the rationals is not Dedekind complete. A chain is Dedekind complete if its underlying linear order is Dedekind complete.

The fundamental theorem of Kamp's states that $T L$ (Until, Since) is expressively equivalent to FOMLO over Dedekind complete chains.

Theorem 2.1 (Kamp [8]). (1) Given any TL(Until, Since) formula $A$ there is a FOMLO formula $\varphi_{A}(x)$ which is equivalent to $A$ over all chains. 
(2) Given any FOMLO formula $\varphi(x)$ with one free variable, there is a TL(Until, Since) formula which is equivalent to $\varphi$ over Dedekind complete chains.

The meaning preserving translation from $T L$ (Until, Since) to FOMLO is easily obtained by structural induction. The contribution of our paper is a proof of Theorem 2.1 (2). The proof is constructive. An algorithm which for every FOMLO formula $\varphi(x)$ constructs a $T L$ (Until, Since) formula which is equivalent to $\varphi$ over Dedekind complete chains is easily extracted from our proof. However, this algorithms is not efficient in the sense of complexity theory. This is unavoidable because there is a non-elementary succinctness gap between FOMLO and $T L$ (Until, Since) even over the class of finite chains, i.e., for every $m, n \in$ $\mathbb{N}$ there is a FOMLO formula $\varphi\left(x_{0}\right)$ of size $|\varphi|>n$ which is not equivalent (even over finite chains) to any $T L$ (Until, Since) formula of $\operatorname{size} \leq \exp (m,|\varphi|)$, where the $m$-iterated exponential function $\exp (m, n)$ is defined by induction on $m$ so that $\exp (1, n)=2^{n}$, and $\exp (m+1, n)=2^{\exp (m, n)}$.

\section{3. $\vec{\exists} \forall$ FORMULAS}

First, we introduce $\vec{\exists} \forall$ formulas which are instances of the Decomposition formulas of [3]. Definition 3.1 ( $\vec{\exists} \forall$-formulas). Let $\Sigma$ be a set of monadic predicate names. An $\vec{\exists} \forall$-formula over $\Sigma$ is a formula of the form:

$$
\begin{aligned}
\psi\left(z_{0}, \ldots, z_{m}\right) & :=\exists x_{n} \ldots \exists x_{1} \exists x_{0} \\
& \left(\bigwedge_{k=0}^{m} z_{k}=x_{i_{k}}\right) \wedge\left(x_{n}>x_{n-1}>\cdots>x_{1}>x_{0}\right) \text { "ordering of } x_{i} \text { and } z_{j} " \\
& \wedge \bigwedge_{j=0}^{n} \alpha_{j}\left(x_{j}\right) \text { "Each } \alpha_{j} \text { holds at } x_{j} " \\
& \wedge \bigwedge_{j=1}^{n}\left[(\forall y)_{>x_{j-1}}^{<x_{j}} \beta_{j}(y)\right] \text { "Each } \beta_{j} \text { holds along }\left(x_{j-1}, x_{j}\right) " \\
& \wedge(\forall y)_{>x_{n}} \beta_{n+1}(y) \text { " } \beta_{n+1} \text { holds everywhere after } x_{n} " \\
& \wedge(\forall y)^{<x_{0}} \beta_{0}(y) \text { " } \beta_{0} \text { holds everywhere before } x_{0} "
\end{aligned}
$$

with a prefix of $n+1$ existential quantifiers and with all $\alpha_{j}, \beta_{j}$ quantifier free formulas with one variable over $\Sigma$, and $i_{0}, \ldots, i_{m} \in\{0, \ldots, n\}$. ( $\psi$ has $m+1$ free variables $z_{0}, \ldots, z_{m}$ and $n+1$ existential quantifiers, $m+1$ quantifiers are dummy and are introduced just in order to simplify notations.)

It is clear that

\section{Lemma 3.2.}

(1) Conjunction of $\vec{\exists} \forall$-formulas is equivalent to a disjunction of $\vec{\exists} \forall$-formulas.

(2) Every $\vec{\exists} \forall$-formula is equivalent to a conjunction of $\vec{\exists} \forall$-formulas with at most two free variables.

(3) For every $\vec{\exists} \forall$-formula $\varphi$ the formula $\exists x \varphi$ is equivalent to a $\vec{\exists} \forall$-formula.

Definition 3.3 ( $\vee \vec{\exists} \forall$-formulas). A formula is a $\vee \vec{\exists} \forall$ formula if it is equivalent to a disjunction of $\vec{\exists} \forall$-formulas. 
Lemma 3.4 (closure properties). The set of $\vee \vec{\exists} \forall$ formulas is closed under disjunction, conjunction, and existential quantification.

Proof. By (1) and (3) of Lemma 3.2, and distributivity of $\exists$ over $\vee$.

The set of $\vee \vec{\exists} \forall$ formulas is not closed under negation ${ }^{2}$. However, we show later (see Proposition 4.3) that the negation of a $\vee \vec{\exists} \forall$ formula is equivalent to a $\vee \vec{\exists} \forall$ formula in the expansion of the chains by all $T L$ (Until, Since) definable predicates.

The $\vee \vec{\exists} \forall$ formulas with one free variable can be easily translated to $T L$ (Until, Since).

Proposition 3.5 (From $\vee \vec{\exists} \forall$-formulas to $T L$ (Until, Since) formulas). Every $\vee \vec{\exists} \forall$-formula with one free variable is equivalent to a TL(Until, Since) formula.

Proof. By a simple formalization we show that every $\vec{\exists} \forall$-formula with one free variable is equivalent to a $T L($ Until, Since) formula. This immediately implies the proposition.

Let $\psi\left(z_{0}\right)$ be an $\vec{\exists} \forall$-formula

$$
\begin{gathered}
\exists x_{n} \ldots \exists x_{1} \exists x_{0} z_{0}=x_{k} \wedge\left(x_{n}>x_{n-1}>\cdots>x_{1}>x_{0}\right) \wedge \bigwedge_{j=0}^{n} \alpha_{j}\left(x_{j}\right) \\
\wedge \bigwedge_{j=1}^{n}(\forall y)_{>x_{j-1}}^{<x_{j}} \beta_{j}(y) \wedge(\forall y)^{<x_{0}} \beta_{0}(y) \wedge(\forall y)_{>x_{n}} \beta_{n+1}(y)
\end{gathered}
$$

Let $A_{i}$ and $B_{i}$ be temporal formulas equivalent to $\alpha_{i}$ and $\beta_{i}\left(A_{i}\right.$ and $B_{i}$ do not even use Until and Since modalities). It is easy to see that $\psi$ is equivalent to the conjunction of

$$
A_{k} \wedge\left(B_{k+1} \operatorname{Until}\left(A_{k+1} \wedge\left(B_{k+2} \operatorname{Until} \cdots\left(A_{n-1} \wedge\left(B_{n} \operatorname{Until}\left(A_{n} \wedge \square B_{n+1}\right)\right) \cdots\right)\right)\right)\right)
$$

and

$$
A_{k} \wedge\left(B _ { k - 1 } \operatorname { S i n c e } \left(A_{k-1} \wedge\left(B_{k-2} \operatorname{Since}\left(\cdots A_{1} \wedge\left(B_{1} \operatorname{Since}\left(A_{0} \wedge \overleftarrow{\square} B_{0}\right)\right) \cdots\right)\right)\right.\right.
$$

\section{Proof of Kamp's TheOREM}

The next definition plays a major role in the proof Kamp's theorem [3].

Definition 4.1. Let $\mathcal{M}$ be a $\Sigma$ chain. We denote by $\mathcal{E}[\Sigma]$ the set of unary predicate names $\Sigma \cup\{A \mid A$ is an $T L($ Until, Since)-formula over $\Sigma\}$. The canonical $T L($ Until, Since)expansion of $\mathcal{M}$ is an expansion of $\mathcal{M}$ to an $\mathcal{E}[\Sigma]$-chain, where each predicate name $A \in \mathcal{E}[\Sigma]$ is interpreted as $\{a \in \mathcal{M} \mid \mathcal{M}, a \models A\}$. We say that first-order formulas in the signature $\mathcal{E}[\Sigma] \cup\{<\}$ are equivalent over $\mathcal{M}$ (respectively, over a class of $\Sigma$-chains $\mathcal{C}$ ) if they are equivalent in the canonical expansion of $\mathcal{M}$ (in the canonical expansion of every $\mathcal{M} \in \mathcal{C}$ ).

\footnotetext{
${ }^{2}$ The truth table of $P$ Until $Q$ is an $\vec{\exists} \forall$ formula $\left(\exists x^{\prime}\right)_{>x}\left(Q\left(x^{\prime}\right) \wedge(\forall y)_{>x}^{<x^{\prime}} P(y)\right)$, yet we can prove that its negation is not equivalent to any $\vee \vec{\exists} \forall$ formula.

${ }^{3}$ We often use " $a \in \mathcal{M}$ " instead of " $a$ is an element of the domain of $\mathcal{M}$ "
} 
Note that if $A$ is a $T L($ Until, Since) formula over $\mathcal{E}[\Sigma]$ predicates, then it is equivalent to a $T L$ (Until, Since) formula over $\Sigma$, and hence to an atomic formula in the canonical $T L($ Until, Since)-expansions.

In this section and the next one we say that "formulas are equivalent in a chain $\mathcal{M}$ " instead of "formulas are equivalent in the canonical $T L$ (Until, Since)-expansion of $\mathcal{M}$." The $\vec{\exists} \forall$ and $\vee \vec{\exists} \forall$ formulas are defined as previously, but now they can use as atoms $T L($ Until, Since) definable predicates.

It is clear that all the results stated above hold for this modified notion of $\vee \vec{\exists} \forall$ formulas. In particular, every $\vee \vec{\exists} \forall$ formula with one free variable is equivalent to an $T L$ (Until, Since) formula, and the set of $\vee \vec{\exists} \forall$ formulas is closed under conjunction, disjunction and existential quantification. However, now the set of $\vee \vec{\exists} \forall$ formulas is also closed under negation, due to the next proposition whose proof is postponed to Sect. 5 .

Proposition 4.2. (Closure under negation) The negation of $\vec{\exists} \forall$-formulas with at most two free variables is equivalent over Dedekind complete chains to a disjunction of $\vec{\exists} \forall$-formulas.

As a consequence we obtain

Proposition 4.3. Every first-order formula is equivalent over Dedekind complete chains to a disjunction of $\vec{\exists} \forall$-formulas.

Proof. We proceed by structural induction.

Atomic: It is clear that every atomic formula is equivalent to a disjunction of (even quantifier free) $\vec{\exists} \forall$-formulas.

Disjunction: - immediate.

Negation: If $\varphi$ is an $\vec{\exists} \forall$-formula, then by Lemma 3.2 (2) it is equivalent to a conjunction of $\vec{\exists} \forall$ formulas with at most two free variables. Hence, $\neg \varphi$ is equivalent to a disjunction of $\neg \psi_{i}$ where $\psi_{i}$ are $\vec{\exists} \forall$-formulas with at most two free variables. By Proposition 4.2, $\neg \psi_{i}$ is equivalent to a disjunction of $\exists \forall$ formulas $\gamma_{i}^{j}$. Hence, $\neg \varphi$ is equivalent to a disjunction $\vee_{i} \vee_{j} \gamma_{i}^{j}$ of $\vec{\exists} \forall$ formulas.

If $\varphi$ is a disjunction of $\vec{\exists} \forall$ formulas $\varphi_{i}$, then $\neg \varphi$ is equivalent to the conjunction of $\neg \varphi_{i}$. By the above, $\neg \varphi_{i}$ is equivalent to a $\vee \vec{\exists} \forall$ formula. Since, $\vee \vec{\exists} \forall$ formulas are closed under conjunction (Lemma 3.4 ), we obtain that $\neg \varphi$ is equivalent to a disjunction of $\vec{\exists} \forall$ formulas.

$\exists$-quantifier: For $\exists$-quantifier, the claim follows from Lemma 3.4.

Now, we are ready to prove Kamp's Theorem:

Theorem 4.4. For every FOMLO formula $\varphi(x)$ with one free variable, a TL(Until, Since) formula exists that is equivalent to $\varphi$ over Dedekind complete chains.

Proof. By Proposition 4.3, $\varphi(x)$ is equivalent over Dedekind complete chains to a disjunction of $\vec{\exists} \forall$ formulas $\varphi_{i}(x)$. By Proposition 3.5, $\varphi_{i}(x)$ is equivalent to a $T L$ (Until, Since) formula. Hence, $\varphi(x)$ is equivalent over Dedekind complete chains to a $T L$ (Until, Since) formula.

This completes our proof of Kamp's theorem except Proposition 4.2 which is proved in the next section. 


\section{Proof of Proposition 4.2}

Let $\psi\left(z_{0}, z_{1}\right)$ be an $\vec{\exists} \forall$-formula

$$
\begin{gathered}
\exists x_{n} \ldots \exists x_{1} \exists x_{0}\left[z_{0}=x_{m} \wedge z_{1}=x_{k} \wedge\left(x_{0}<x_{1}<\cdots<x_{n-1}<x_{n}\right) \wedge \bigwedge_{j=0}^{n} \alpha_{j}\left(x_{j}\right)\right. \\
\left.\wedge \bigwedge_{j=1}^{n}(\forall y)_{>x_{j-1}}^{<x_{j}} \beta_{j}(y) \wedge(\forall y)^{<x_{0}} \beta_{0}(y) \wedge(\forall y)_{>x_{n}} \beta_{n+1}(y)\right]
\end{gathered}
$$

We consider two cases. In the first case $k=m$, i.e., $z_{0}=z_{1}$ and in the second $k \neq m$.

If $k=m$, then $\psi$ is equivalent to $z_{0}=z_{1} \wedge \psi^{\prime}\left(z_{0}\right)$, where $\psi^{\prime}$ is an $\vec{\exists} \forall$-formula. By Proposition 3.5, $\psi^{\prime}$ is equivalent to an $T L$ (Until, Since) formula $A^{\prime}$. Therefore, $\psi$ is equivalent to an $\vec{\exists} \forall$-formula $\exists x_{0}\left[z_{0}=x_{0} \wedge z_{1}=x_{0} \wedge A^{\prime}\left(x_{0}\right)\right]$, and $\neg \psi$ is equivalent to a $\vee \vec{\exists} \forall$ formula $z_{0}<z_{1} \vee z_{1}<z_{0} \vee \exists x_{0}\left[z_{0}=x_{0} \wedge z_{1}=x_{0} \wedge \neg A^{\prime}\left(x_{0}\right)\right]$

If $k \neq m$, w.l.o.g. we assume that $m<k$. Hence, $\psi$ is equivalent to a conjunction of

(1) $\psi_{0}\left(z_{0}\right)$ defined as:

$$
\begin{aligned}
\exists x_{0} \ldots \exists x_{m-1} \exists x_{m}\left[z_{0}=\right. & x_{m} \wedge\left(x_{0}<x_{1}<\cdots<x_{m}\right) \wedge \bigwedge_{j=0}^{m} \alpha_{j}\left(x_{j}\right) \\
& \left.\wedge \bigwedge_{j=1}^{m}(\forall y)_{>x_{j-1}}^{<x_{j}} \beta_{j}(y) \wedge(\forall y)^{<x_{0}} \beta_{0}(y)\right]
\end{aligned}
$$

(2) $\psi_{1}\left(z_{1}\right)$ defined as:

$$
\begin{aligned}
\exists x_{k} \ldots \exists x_{k+1} \exists x_{n}\left[z_{1}\right. & =x_{k} \wedge\left(x_{k}<x_{k+1}<\cdots<x_{n}\right) \wedge \bigwedge_{j=k}^{n} \alpha_{j}\left(x_{j}\right) \\
& \left.\wedge \bigwedge_{j=k+1}^{n}(\forall y)_{>x_{j-1}}^{<x_{j}} \beta_{j}(y) \wedge(\forall y)_{>x_{n}} \beta_{n+1}(y)\right]
\end{aligned}
$$

(3) $\varphi\left(z_{0}, z_{1}\right)$ defined as:

$$
\begin{aligned}
\exists x_{m} \ldots \exists x_{k}\left[\left(z_{0}=x_{m}<x_{m+1}\right.\right. & \left.<\cdots<x_{k}=z_{1}\right) \wedge \bigwedge_{j=m}^{k} \alpha_{j}\left(x_{j}\right) \\
& \left.\wedge \bigwedge_{j=m+1}^{k}(\forall y)_{>x_{j-1}}^{<x_{j}} \beta_{j}(y)\right]
\end{aligned}
$$

The first two formulas are $\vec{\exists} \forall$-formulas with one free variable. Therefore, (by Proposition 3.5) they are equivalent to $T L($ Until, Since) formulas (in the signature $\mathcal{E}[\Sigma]$ ). Hence, their negations are equivalent (over the canonical expansions) to atomic (and hence to $\vec{\exists} \forall$ ) formulas.

Therefore, it is sufficient to show that the negation of the third formula is equivalent over Dedekind complete chains to a disjunction of $\vec{\exists} \forall$-formulas. This is stated in the following lemma:

Lemma 5.1. The negation of any formula of the form

$$
\exists x_{0} \ldots \exists x_{n}\left[\left(z_{0}=x_{0}<\cdots<x_{n}=z_{1}\right) \wedge \bigwedge_{j=0}^{n} \alpha_{j}\left(x_{j}\right) \wedge \bigwedge_{j=1}^{n}(\forall y)_{>x_{j-1}}^{<x_{j}} \beta_{j}(y)\right]
$$

where $\alpha_{i}, \beta_{i}$ are quantifier free, is equivalent (over Dedekind complete chains) to a disjunction of $\vec{\exists} \forall$-formulas. 
In the rest of this section we prove Lemma 5.1. Our proof is organized as follows. In Lemma 5.3 we prove an instance of Lemma 5.1 where $\alpha_{0}, \alpha_{n}$ and all $\beta_{i}$ are equivalent to True. Then we derive a more general instance (Corollary 5.4) where $\beta_{n}$ is equivalent to true. Finally we prove the full version of Lemma 5.1 .

First, we introduce some helpful notations.

Notation 5.2. We use the abbreviated notation $\left[\alpha_{0}, \beta_{1}, \ldots, \alpha_{n-1}, \beta_{n}, \alpha_{n}\right]\left(z_{0}, z_{1}\right)$ for the $\vec{\exists} \forall$-formula as in (5.1).

In this notation Lemma 5.1 can be rephrased as $\neg\left[\alpha_{0}, \beta_{1}, \ldots, \alpha_{n-1}, \beta_{n}, \alpha_{n}\right]\left(z_{0}, z_{1}\right)$ is equivalent (over Dedekind complete chains) to a $\vee \vec{\exists} \forall$ formula.

We start with the instance of Lemma 5.1 where all $\beta_{i}$ are True.

Lemma 5.3. $\neg \exists x_{1} \ldots \exists x_{n}\left(z_{0}<x_{1}<\cdots<x_{n}<z_{1}\right) \wedge \bigwedge_{i=1}^{n} P_{i}\left(x_{i}\right)$ is equivalent over Dedekind complete chains to $a \vee \vec{\exists} \forall$ formula $O_{n}\left(P_{1}, \ldots, P_{n}, z_{0}, z_{1}\right)$.

Proof. We proceed by induction on $n$.

Basis: $\neg\left(\exists x_{1}\right)_{>z_{0}}^{<z_{1}} P_{1}\left(x_{1}\right)$ is equivalent to $(\forall y)_{>z_{0}}^{<z_{1}} \neg P_{1}(y)$.

Inductive step: $n \mapsto n+1$. We assume that a $\vee \vec{\exists} \forall$ formula $O_{n}$ has already defined and construct a $\vee \vec{\exists} \forall$ formula $O_{n+1}$.

Observe that if the interval $\left(z_{0}, z_{1}\right)$ is non-empty, then one of the following cases holds:

Case 1: $P_{1}$ does not occur in $\left(z_{0}, z_{1}\right)$, i.e. $(\forall y)_{>z_{0}}^{<z_{1}} \neg P_{1}(y)$. Then $O_{n+1}\left(P_{1}, \ldots, P_{n+1}, z_{0}, z_{1}\right)$ should be equivalent to True.

Case 2: If case 1 does not hold then let $r_{0}=\inf \left\{z \in\left(z_{0}, z_{1}\right) \mid P_{1}(z)\right\}$ (such $r_{0}$ exists by Dedekind completeness. Note that $r_{0}=z_{0}$ iff $\mathbf{K}^{+}\left(P_{1}\right)\left(z_{0}\right)$. If $r_{0}>z_{0}$ then $r_{0} \in\left(z_{0}, z_{1}\right)$ and $r_{0}$ is definable by the following $\vee \vec{\exists} \forall$ formula:

$$
\begin{aligned}
\operatorname{INF}\left(z_{0}, r_{0}, z_{1}, P_{1}\right):=z_{0} & <r_{0}<z_{1} \wedge(\forall y)_{>z_{0}}^{<r_{0}} \neg P_{1}(y) \wedge \\
& \wedge\left(P_{1}\left(r_{0}\right) \vee \mathbf{K}^{+}\left(P_{1}\right)\left(r_{0}\right)\right)
\end{aligned}
$$

Subcase $r_{0}=z_{0}$ : In this subcase $O_{n}\left(P_{2}, \ldots, P_{n}, z_{0}, z_{1}\right)$ and $O_{n+1}\left(P_{1}, \ldots, P_{n+1}, z_{0}, z_{1}\right)$ should be equivalent.

Subcase $r_{0} \in\left(z_{0}, z_{1}\right):$ Now $O_{n}\left(P_{2}, \ldots, P_{n}, r_{0}, z_{1}\right)$ and $O_{n+1}\left(P_{1}, \ldots, P_{n+1}, z_{0}, z_{1}\right)$ should be equivalent.

Hence, $O_{n+1}\left(P_{1}, \ldots, P_{n+1}, z_{0}, z_{1}\right)$ can be defined as the disjunction of " $\left(z_{0}, z_{1}\right)$ is empty" and the following formulas:

(1) $(\forall y)_{>z_{0}}^{<z_{1}} \neg P_{1}((y)$

(2) $\mathbf{K}^{+}\left(P_{1}\right)\left(z_{0}\right) \wedge O_{n}\left(P_{2}, \ldots, P_{n}, z_{0}, z_{1}\right)$

(3) $\left(\exists r_{0}\right)_{>z_{0}}^{<z_{1}}\left(\operatorname{INF}\left(z_{0}, r_{0}, z_{1}, P_{1}\right) \wedge O_{n}\left(P_{2}, \ldots, P_{n}, r_{0}, z_{1}\right)\right)$

The first formula is a $\vee \vec{\exists} \forall$ formula. By the inductive assumptions $O_{n}$ is a $\vee \vec{\exists} \forall$ formula. $\mathbf{K}^{+}\left(P_{1}\right)\left(z_{0}\right)$ is an atomic (and hence a $\vee \vec{\exists} \forall$ ) formula in the canonical expansion, and $\operatorname{INF}\left(z_{0}, r_{0}, z_{1}, P_{1}\right)$ is a $\vee \vec{\exists} \forall$ formula. Since $\vee \vec{\exists} \forall$ formulas are closed under conjunction, disjunction and the existential quantification, we conclude that $O_{n+1}$ is a $\vee \vec{\exists} \forall$ formula.

As a consequence we obtain 
Corollary 5.4.

(1) $\neg(\exists z)_{>z_{0}}^{<z_{1}}\left[\alpha_{0}, \beta_{1}, \alpha_{1}, \beta_{2}, \ldots, \alpha_{n-1}, \beta_{n}, \alpha_{n}\right]\left(z_{0}, z\right)$ over Dedekind complete chains is equivalent to $a \vee \vec{\exists} \forall$ formula.

(2) $\neg(\exists z)_{>z_{0}}^{<z_{1}}\left[\alpha_{0}, \beta_{1}, \alpha_{1}, \beta_{2}, \ldots, \alpha_{n-1}, \beta_{n}, \alpha_{n}\right]\left(z, z_{1}\right)$ over Dedekind complete chains is equivalent to $a \vee \vec{\exists} \forall$ formula.

Proof. (1) Define

$$
\begin{array}{r}
F_{n}:=\alpha_{n} \\
F_{i-1}:=\alpha_{i-1} \wedge\left(\beta_{i} \text { Until } F_{i}\right) \quad \text { for } i=1, \ldots, n
\end{array}
$$

Observe that there is $z \in\left(z_{0}, z_{1}\right)$ such that $\left[\alpha_{0}, \beta_{1}, \alpha_{1}, \beta_{2}, \ldots, \alpha_{n-1}, \beta_{n}, \alpha_{n}\right]\left(z_{0}, z\right)$ iff $F_{0}\left(z_{0}\right)$ and there is an increasing sequence $x_{1}<\cdots<x_{n}$ in an open interval $\left(z_{0}, z_{1}\right)$ such that $F_{i}\left(x_{i}\right)$ for $i=1, \ldots, n$. Indeed, the direction $\Rightarrow$ is trivial. The direction $\Leftarrow$ is easily proved by induction.

The basis is trivial.

Inductive step: $n \mapsto n+1$. Assume $F_{0}\left(z_{0}\right)$ holds and that $\left(z_{0}, z_{1}\right)$ contains an increasing sequence $x_{1}<\cdots<x_{n+1}$ such that $F_{i}\left(x_{i}\right)$ for $i=1, \ldots, n+1$. By the inductive assumption there is $y_{1} \in\left(z_{0}, x_{n+1}\right)$ such that

$$
\left[\alpha_{0}, \beta_{1}, \alpha_{1}, \beta_{2}, \ldots, \beta_{n-1} \alpha_{n-1}, \beta_{n},\left(\alpha_{n} \wedge \beta_{n+1} \text { Until } \alpha_{n+1}\right)\right]\left(z_{0}, y_{1}\right) .
$$

In particular, $y_{1}$ satisfies $\left(\alpha_{n} \wedge \beta_{n+1}\right.$ Until $\left.\alpha_{n+1}\right)$. Hence, there is $y_{2}>y_{1}$ such that $y_{2}$ satisfies $\alpha_{n+1}$ and $\beta_{n+1}$ holds along $\left(y_{1}, y_{2}\right)$.

If $y_{2} \leq x_{n+1}$ then the required $z \in\left(z_{0}, z_{1}\right)$ equals to $y_{2}$, and we are done. Otherwise, $x_{n+1}<y_{2}$. Therefore, $x_{n+1} \in\left(y_{1}, y_{2}\right)$ and $\beta_{n+1}$ holds along $\left(y_{1}, x_{n+1}\right)$. Hence, the required $z$ equals to $x_{n+1}$.

The above observation and Lemma 5.3 imply that $\neg F_{0}\left(z_{0}\right) \vee O_{n}\left(F_{1}, \ldots, F_{n}, z_{0}, z_{1}\right)$ is a $\vee \vec{\exists} \forall$ formula that is equivalent to $\neg(\exists z)_{>z_{0}}^{<z_{1}}\left[\alpha_{0}, \beta_{1}, \alpha_{1}, \beta_{2}, \ldots, \alpha_{n-1}, \beta_{n}, \alpha_{n}\right]\left(z_{0}, z\right)$.

$(2)$ is the mirror image of (1) and is proved similarly.

Now we are ready to prove Lemma [5.1, i.e.,

$$
\begin{aligned}
& \neg\left[\alpha_{0}, \beta_{1} \ldots, \beta_{n-1}, \alpha_{n-1}, \beta_{n}, \alpha_{n}\right]\left(z_{0}, z_{1}\right) \text { is equivalent } \\
& \text { over Dedekind complete chains to a } \vee \vec{\exists} \forall \text { formula. }
\end{aligned}
$$

Proof. (of Lemma 5.1) If the interval $\left(z_{0}, z_{1}\right)$ is empty then the assertion is immediate. We assume that $\left(z_{0}, z_{1}\right)$ is non-empty. Hence, at least one of the following cases holds:

Case 1: $\neg \alpha_{0}\left(z_{0}\right)$ or $\mathbf{K}^{+}\left(\neg \beta_{1}\right)\left(z_{0}\right)$.

Case 2: $\alpha_{0}\left(z_{0}\right)$, and $\beta_{1}$ holds along $\left(z_{0}, z_{1}\right)$.

Case 3: (1) $\alpha_{0}\left(z_{0}\right) \wedge \neg \mathbf{K}^{+}\left(\neg \beta_{1}\right)\left(z_{0}\right)$, and

(2) there is $x \in\left(z_{0}, z_{1}\right)$ such that $\neg \beta_{1}(x)$.

For each of these cases we construct a $\vee \vec{\exists} \forall$ formula Cond $_{i}$ that describes it (i.e., Case $i$ holds iff Cond $_{i}$ holds) and show that if Cond $_{i}$ holds, then $\neg\left[\alpha_{0}, \beta_{1} \ldots, \beta_{n-1}, \alpha_{n-1}, \beta_{n}, \alpha_{n}\right]\left(z_{0}, z_{1}\right)$

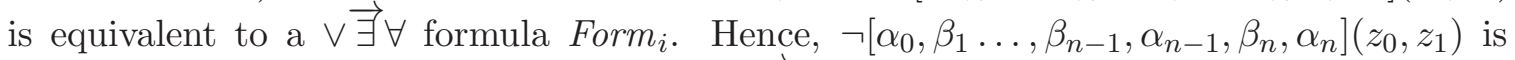
equivalent to $\vee_{i}\left[\right.$ Cond $_{i} \wedge$ Form $\left._{i}\right]$ which is a $\vee \vec{\exists} \forall$ formula.

Case 1 This case is already explicitly described by the $\vee \vec{\exists} \forall$ formula (in the canonical expansion). In this case $\neg\left[\alpha_{0}, \beta_{1} \ldots, \beta_{n-1}, \alpha_{n-1}, \beta_{n}, \alpha_{n}\right]\left(z_{0}, z_{1}\right)$ is equivalent to True. 


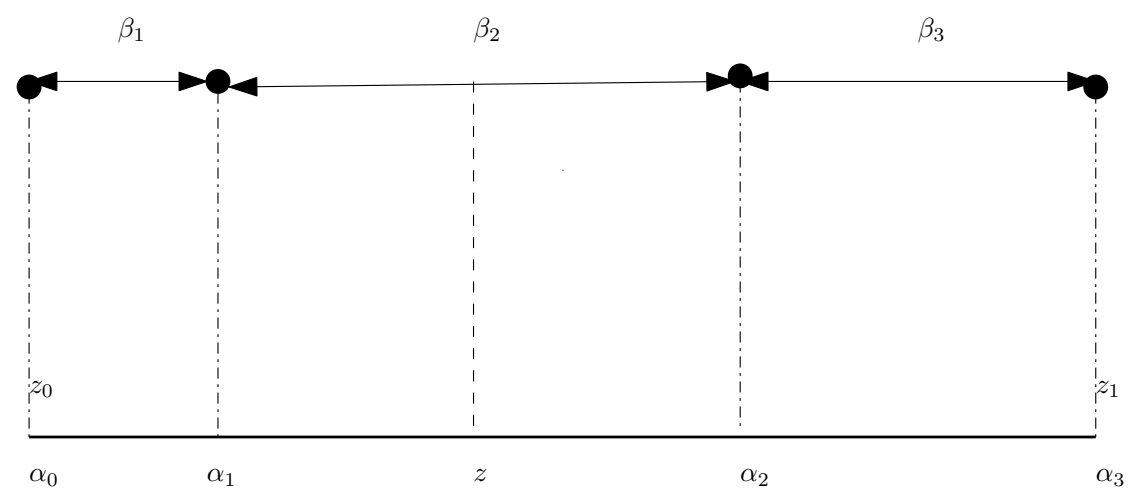

Figure 1. $B_{2}\left(z_{0}, z, z_{1}\right):=\left[\alpha_{0}, \beta_{1}, \alpha_{1}, \beta_{2}, \beta_{2}\right]\left(z_{0}, z\right) \wedge\left[\beta_{2}, \beta_{2}, \alpha_{2}, \beta_{3}, \alpha_{3}\right]\left(z, z_{1}\right)$

Case 2 This case is described by a $\vee \vec{\exists} \forall$ formula $\alpha_{0}\left(z_{0}\right) \wedge(\forall z)_{>z_{0}}^{<z_{1}} \beta_{1}$. In this case $\neg\left[\alpha_{0}, \beta_{1} \ldots, \beta_{n-1}, \alpha_{n-1}, \beta_{n}, \alpha_{n}\right]\left(z_{0}, z_{1}\right)$ is equivalent to "there is no $z \in\left(z_{0}, z_{1}\right)$ such that $\left[\alpha_{1}, \beta_{2} \ldots, \beta_{n}, \alpha_{n}\right]\left(z, z_{1}\right)$." By Corollary [5.4 (2) this is expressible by a $\vee \vec{\exists} \forall$ formula.

Case 3 The first condition of Case 3 is already explicitly described by a $\vee \vec{\exists} \forall$ formula. When the first condition holds, then the second condition is equivalent to "there is (a unique) $r_{0} \in\left(z_{0}, z_{1}\right)$ such that $r_{0}=\inf \left\{z \in\left(z_{0}, z_{1}\right) \mid \neg \beta_{1}(z)\right\} "$ (If $\neg \mathbf{K}^{+}\left(\neg \beta_{1}\right)$ holds at $z_{0}$ and there is $x \in\left(z_{0}, z_{1}\right)$ such that $\neg \beta_{1}(x)$, then such $r_{0}$ exists because we deal with Dedekind complete chains.) This $r_{0}$ is definable by the following $\vee \vec{\exists} \forall$ formula, i.e., it is a unique $z$ which satisfies it:

$$
I N F^{\neg \beta_{1}}\left(z_{0}, z, z_{1}\right):=z_{0}<z<z_{1} \wedge(\forall y)_{>z_{0}}^{<z} \beta_{1}(y) \wedge\left(\neg \beta_{1}(z) \vee \mathbf{K}^{+}\left(\neg \beta_{1}\right)(z)\right)
$$

Hence, Case 3 is described by $\alpha_{0}\left(z_{0}\right) \wedge \neg \mathbf{K}^{+}\left(\neg \beta_{1}\right)\left(z_{0}\right) \wedge(\exists z)_{>z_{0}}^{<z_{1}} I N F^{\neg \beta_{1}}\left(z_{0}, z, z_{1}\right)$ which is equivalent to an $\vec{\exists} \forall$ formula.

It is sufficient to show that $(\exists z)_{>z_{0}}^{<z_{1}} I N F^{\neg \beta_{1}}(z) \wedge \neg\left[\alpha_{0}, \beta_{1}, \alpha_{1}, \ldots, \beta_{n+1}, \alpha_{n+1}\right]\left(z_{0}, z_{1}\right)$ is equivalent to a $\vee \vec{\exists} \forall$ formula.

We prove this by induction on $n$.

The basis is trivial.

Inductive step $n \mapsto n+1$.

Define:

$$
\begin{aligned}
A_{i}^{-}\left(z_{0}, z\right) & :=\left[\alpha_{0}, \beta_{1}, \ldots, \beta_{i}, \alpha_{i}\right]\left(z_{0}, z\right) & i=1, \ldots, n \\
A_{i}^{+}\left(z, z_{1}\right) & :=\left[\alpha_{i}, \beta_{i+1}, \ldots \beta_{n+1}, \alpha_{n+1}\right]\left(z, z_{1}\right) & i=1, \ldots, n \\
A_{i}\left(z_{0}, z, z_{1}\right) & :=A_{i}^{-}\left(z_{0}, z\right) \wedge A_{i}^{+}\left(z, z_{1}\right) & i=1, \ldots, n \\
B_{i}^{-}\left(z_{0}, z\right) & :=\left[\alpha_{0}, \beta_{1}, \ldots, \beta_{i-1}, \alpha_{i-1}, \beta_{i}, \beta_{i}\right]\left(z_{0}, z\right) & i=1, \ldots, n+1 \\
B_{i}^{+}\left(z, z_{1}\right) & :=\left[\beta_{i}, \beta_{i}, \alpha_{i} \beta_{i+1} \alpha_{i+1}, \ldots, \beta_{n+1}, \alpha_{n+1}\right]\left(z, z_{1}\right) & i=1, \ldots, n+1 \\
B_{i}\left(z_{0}, z, z_{1}\right) & :=B_{i}^{-}\left(z_{0}, z\right) \wedge B_{i}^{+}\left(z, z_{1}\right) & i=1, \ldots, n+1
\end{aligned}
$$

\footnotetext{
${ }^{4}$ We will use only existence and will not use uniqueness.
} 
If the interval $\left(z_{0}, z_{1}\right)$ is non-empty, these definitions imply

$$
\begin{aligned}
{\left[\alpha_{0}, \beta_{1}, \alpha_{1}, \ldots, \beta_{n+1}, \alpha_{n+1}\right]\left(z_{0}, z_{1}\right) \Leftrightarrow(\forall z)_{>z_{0}}^{<z_{1}}\left(\bigvee_{i=1}^{n} A_{i} \vee \bigvee_{i=1}^{n+1} B_{i}\right) } \\
{\left[\alpha_{0}, \beta_{1}, \alpha_{1}, \ldots, \beta_{n+1}, \alpha_{n+1}\right]\left(z_{0}, z_{1}\right) \Leftrightarrow(\exists z)_{>z_{0}}^{<z_{1}}\left(\bigvee_{i=1}^{n} A_{i} \vee \bigvee_{i=1}^{n+1} B_{i}\right) }
\end{aligned}
$$

Hence, for every $\varphi$

$$
(\exists z)_{>z_{0}}^{<z_{1}} \varphi(z) \wedge \neg\left[\alpha_{0}, \beta_{1}, \alpha_{1}, \ldots, \beta_{n+1}, \alpha_{n+1}\right]\left(z_{0}, z_{1}\right)
$$

is equivalent to

$$
(\exists z)_{>z_{0}}^{<z_{1}}\left(\varphi(z) \wedge \bigwedge_{i=1}^{n} \neg A_{i} \wedge \bigwedge_{i=1}^{n+1} \neg B_{i}\right)
$$

In particular,

$$
(\exists z)_{>z_{0}}^{<z_{1}} I N F^{\neg \beta_{1}}(z) \wedge \neg\left[\alpha_{0}, \beta_{1}, \alpha_{1}, \ldots, \beta_{n+1}, \alpha_{n+1}\right]\left(z_{0}, z_{1}\right)
$$

is equivalent to

$$
(\exists z)_{>z_{0}}^{<z_{1}}\left(I N F \neg \beta_{1}(z) \wedge \bigwedge_{i=1}^{n} \neg A_{i} \wedge \bigwedge_{i=1}^{n+1} \neg B_{i}\right),
$$

where $I N F^{\neg \beta_{1}}(z)$ was defined in equation (5.3).

By the inductive assumption

(a): $\neg A_{i}$ is equivalent to a $\vee \vec{\exists} \forall$ formula for $i=1, \ldots, n$.

(b): $\neg B_{i}$ is equivalent to a $\vee \vec{\exists} \forall$ formula for $i=2, \ldots, n$.

Recall $B_{1}:=B_{1}^{-} \wedge B_{1}^{+}$and $B_{n+1}:=B_{n+1}^{-} \wedge B_{n+1}^{+}$.

(c): $\neg B_{1}^{-}$and $\neg B_{n+1}^{+}$are equivalent to $\vee \vec{\exists} \forall$ formulas, by the induction basis.

(d): $I N F \neg \beta_{1}(z) \wedge \neg B_{1}^{+}\left(z, z_{1}\right)$ is equivalent to $I N F \neg \beta_{1}(z)$, because if $I N F \neg \beta_{1}(z)$, then for no $x>z, \beta_{1}$ holds along $[z, x)$.

(e): $\operatorname{INF}^{\neg \beta_{1}}(z) \wedge \neg B_{n+1}^{-}\left(z_{0}, z\right)$ is equivalent to $\operatorname{INF}^{\neg \beta_{1}}(z) \wedge$ (" $\beta_{1}$ holds on $\left(z_{0}, z\right) " \wedge$ $\left.\neg B_{n+1}^{-}\left(z_{0}, z\right)\right)$. Since, by case 2 , " $\beta_{1}$ holds on $\left(z_{0}, z\right)$ " $\wedge \neg B_{n+1}^{-}\left(z_{0}, z\right)$ is equivalent to a $\vee \vec{\exists} \forall$ formula, and $I N F^{\neg \beta_{1}}(z)$ is a $\vee \vec{\exists} \forall$ formula, we conclude that $\operatorname{INF}^{\neg \beta_{1}}(z) \wedge$ $\neg B_{n+1}^{-}\left(z_{0}, z\right)$ is equivalent to a $\vee \vec{\exists} \forall$ formula.

Since the set of $\vee \vec{\exists} \forall$ formulas is closed under conjunction, disjunction and $\exists$, by (a)-(e) we obtain that $(\exists z)_{>z_{0}}^{<z_{1}}\left(I N F^{\neg \beta_{1}}(z) \wedge \bigwedge_{i=1}^{n} \neg A_{i} \wedge \bigwedge_{i=1}^{n+1} \neg B_{i}\right)$ is equivalent to a $\vee \vec{\exists} \forall$ formula. Therefore, $(\exists z)_{>z_{0}}^{<z_{1}} I N F^{\neg \beta_{1}}(z) \wedge \neg\left[\alpha_{0}, \beta_{1}, \alpha_{1}, \ldots, \beta_{n+1}, \alpha_{n+1}\right]\left(z_{0}, z_{1}\right)$ is also a $\vee \vec{\exists} \forall$ formula.

This completes our proof of Lemma 5.1 and of Proposition 4.2 . 


\section{Related Works}

Kamp's theorem was proved in

(1) Kamp's thesis [8] (proof $>100$ pages).

(2) Outlined by Gabbay, Pnueli, Shelah and Stavi [3] (Sect. 2) for $\mathbb{N}$ and stated that it can be extended to Dedekind complete orders using game arguments.

(3) Was proved by Gabbay [1] by separation arguments for $\mathbb{N}$, and extended to Dedekind complete order in [2].

(4) Was proved by Hodkinson [5] by game arguments and simplified in [6] (unpublished).

A temporal logic has the separation property if its formulas can be equivalently rewritten as a boolean combination of formulas, each of which depends only on the past, present or future. The separation property was introduced by Gabbay [1], and surprisingly, a temporal logic which can express $\square$ and $\overleftarrow{\square}$ has the separation property (over a class $\mathcal{C}$ of structures) iff it is expressively complete for $F O M L O$ over $\mathcal{C}$.

The separation proof for $T L$ (Until, Since) over $\mathbb{N}$ is manageable; however, over the real (and over Dedekind complete) chains it contains many rules and transformations and is not easy to follow. Hodkinson and Reynolds [7] write:

The proofs of theorems 18 and 19 [Kamp's theorem over naturals and over reals, respectively] are direct, showing that each formula can be separated. They are tough and tougher, respectively. Nonetheless, they are effective, and so, whilst not quite providing an algorithm to determine if a set of connectives is expressively complete, they do suggest a potential way of telling in practice whether a given set of connectives is expressively complete - in Gabbay's words, try to separate and see where you get stuck!

The game arguments are easier to grasp, but they use complicated inductive assertions. The proof in [6] proceeds roughly as follows. Let $\mathcal{L}_{r}$ be the set of $T L$ (Until, Since) formulas of nesting depth at most $r$. A formula of the form: $\exists \bar{x} \forall y \chi(\bar{x}, y, \bar{z})$ where $\bar{x}$ is an $n$-tuple of variables and $\chi$ is a quantifier free formula over $\{<,=\}$ and $\mathcal{L}_{r}$-definable monadic predicates is called $\langle n, r\rangle$-decomposition formula. The main inductive assertion is proved by "unusual back-and-forth games" and can be rephrased in logical terms as there is a function $f$ : $\mathbb{N} \rightarrow \mathbb{N}$ such that for every $n, r \in \mathbb{N}$, the negation of positive Boolean combinations $\langle n, r\rangle$ decomposition formula is equivalent to a positive Boolean combination of $\langle f(n),(n+r)\rangle$ decomposition formulas.

Our proof is inspired by [3] and [6]; however, it avoids games, and it separates general logical equivalences and temporal arguments.

The temporal logic with the modalities Until and Since is not expressively complete for FOMLO over the rationals. Stavi introduced two additional modalities Until ${ }^{S}$ and Since ${ }^{s}$ and proved that $T L\left(\right.$ Until, Since, Until ${ }^{s}$, Since $\left.^{s}\right)$ is expressively complete for FOMLO over all linear orders [2. In the forthcoming paper we prove Stavi's theorem. The proof is similar to our proof of Kamp's theorem; however, it treats some additional cases related to gaps in orders, and replaces $\vec{\exists} \forall$-formulas by slightly more general formulas.

\section{Future Fragment of FOMLO}

Many temporal formalisms studied in computer science deal only with future formulas, whose truth value at any moment is determined by what happens from a current moment on. 
A formula (temporal, or monadic with a single free first-order variable) $F$ is (semantically) future if for every chain $\mathcal{M}$ and moment $t_{0} \in \mathcal{M}$ :

$$
\mathcal{M}, t_{0} \models F \text { iff }\left.\mathcal{M}\right|_{\geq t_{0}}, t_{0} \models F,
$$

where $\left.\mathcal{M}\right|_{\geq t_{0}}$ is the subchain of $\mathcal{M}$ over the interval $\left[t_{0}, \infty\right)$. For example, $P$ Until $Q$ and $\mathbf{K}^{+}(P)$ are future formulas, while $P$ Since $Q$ and $\mathbf{K}^{-}(P)$ are not future ones.

For a set $B$ of modalities we denote by $T L(B)$ the temporal logic which uses only modalities from $B$. In particular, $T L($ Until) is the temporal logic which uses the modality Until and $T L\left(\right.$ Until, $\left.\mathbf{K}^{-}\right)$is the temporal logic with modalities Until and $\mathbf{K}^{-}$.

It was shown in [3] that Kamp's theorem holds also for future formulas of FOMLO over $\omega=\langle\mathbb{N},<\rangle$ :

Theorem 7.1 (Gabbay, Pnueli, Shelah, Stavi [3]). Every future FOMLO formula is equivalent over $\omega$-chains to a TL(Until) formula.

The situation is radically different for the continuous time $\langle\mathbb{R},<\rangle$. In [4] it was shown that $T L($ Until) is not expressively complete for the future fragment of FOMLO and there is no easy way to remedy it. In fact, it was shown in [4] that there is no temporal logic with a finite set of modalities which is expressively equivalent to the future fragment of FOMLO over the Reals.

From the separation proof of Kamp's theorem in 2 it follows that every future FOMLO formula is equivalent over Dedekind complete chains to a $T L\left(U n t i l, \mathbf{K}^{-}\right)$formula.

This future-past mixture of Until and $\mathbf{K}^{-}$is somewhat better than the standard Until Since basis in the following sense: although $\mathbf{K}^{-}$is (like Since) a past modality, it does not depend on much of the past. The formula $\mathbf{K}^{-}(P)$ depends just on an arbitrarily short 'near past', and is actually independent of most of the past. In this sense, we may say that it is an "almost" future formula.

Definition 7.2 (Syntactically future $T L\left(\right.$ Until, $\mathbf{K}^{-}$) formulas). A $T L\left(\right.$ Until, $\mathbf{K}^{-}$) formula is syntactically future if it is a boolean combination of atomic formulas and formulas of the form $\varphi_{1}$ Until $\varphi_{2}$, where $\varphi_{1}$ and $\varphi_{2}$ are arbitrary $T L\left(U n t i l, \mathbf{K}^{-}\right)$formulas.

The following lemma immediately follows from the definition and the observation that $\left.\mathcal{M}\right|_{\geq t_{0}}, t_{0} \models \neg \mathbf{K}^{-}(\varphi)$.

Lemma 7.3. A syntactically future $T L\left(\right.$ Until, $\left.\boldsymbol{K}^{-}\right)$formula is future. A $T L\left(\right.$ Until, $\left.\boldsymbol{K}^{-}\right)$formula is future iff it is equivalent to a syntactically future $T L\left(\right.$ Until, $\left.\boldsymbol{K}^{-}\right)$formula.

The next theorem (implicitly) appears in [2] (Chapter 8).

Theorem 7.4. Every future FOMLO formula is equivalent over Dedekind complete chains to a syntactically future $T L\left(\right.$ Until, $\left.\boldsymbol{K}^{-}\right)$formula.

Since $\mathbf{K}^{-} \varphi$ is equivalent to False over discrete orders, we obtain that Theorems 7.1 is an instance of Theorem 7.4 .

Theorem 7.4 is easily obtained by a slight refinement of our proof of Kamp's theorem. We outline its proof in the rest of this section.

Definition $7.5\left(\left(z_{0}, z_{1}\right)-\vec{\exists} \forall\right.$ formula). Let $z_{0}$ and $z_{1}$ be two variables. A formula $z_{0}>z_{1}$, $z_{0}=z_{1}$ or of the form $\left[\alpha_{0}, \beta_{1} \ldots, \beta_{n-1}, \alpha_{n-1}, \beta_{n}, \alpha_{n}\right]\left(z_{0}, z_{1}\right)$ is called a $\left(z_{0}, z_{1}\right)-\vec{\exists} \forall$ formula. A formula is $\left(z_{0}, z_{1}\right)-\vee \vec{\exists} \forall$ formula if it is equivalent to a disjunction of $\left(z_{0}, z_{1}\right)-\vec{\exists} \forall$ formulas. 
Lemma 7.6 (closure properties). The set of $\left(z_{0}, z_{1}\right)-\vee \vec{\exists} \forall$ formulas is closed under disjunction and conjunction. If $\varphi_{1}$ is a $\left(z_{0}, z_{1}\right)-\vee \vec{\exists} \forall$ formula and $\varphi_{2}$ is a $\left(z_{1}, z_{2}\right)-\vee \vec{\exists} \forall$ formula, then $\left(\exists z_{1}\right)_{>z_{0}}^{<z_{2}}\left(\varphi_{1} \wedge \varphi_{2}\right)$ is a $\left(z_{0}, z_{2}\right)-\vee \vec{\exists} \forall$ formula.

The set of $\left(z_{0}, z_{1}\right)-\vee \vec{\exists} \forall$ formulas is not closed under negation. However, we show that the negation of a $\vee \vec{\exists} \forall$ formula is equivalent to a $\left(z_{0}, z_{1}\right)-\vee \vec{\exists} \forall$ formula in the expansion of the chains by all $T L\left(\right.$ Until, $\left.\mathbf{K}^{-}\right)$definable predicates.

Definition 7.7 (The canonical $T L\left(\right.$ Until, $\mathbf{K}^{-}$) and $T L\left(\right.$ Since, $\left.\mathbf{K}^{+}\right)$expansions). Let $\mathcal{M}$ be a $\Sigma$ chain. We denote by $\mathcal{E}\left[\Sigma, T L\left(\right.\right.$ Until, $\left.\left.\mathbf{K}^{-}\right)\right]$the set of unary predicate names $\Sigma \cup\{A$ | $A$ is an $T L\left(\right.$ Until, $\left.\mathbf{K}^{-}\right)$formula over $\left.\Sigma\right\}$. The canonical $T L\left(\right.$ Until, $\left.\mathbf{K}^{-}\right)$-expansion of $\mathcal{M}$ is an expansion of $\mathcal{M}$ to an $\mathcal{E}\left[\Sigma, T L\left(\right.\right.$ Until, $\left.\left.^{-}\right)\right]$-chain, where each predicate's name $A \in$ $\mathcal{E}\left[\Sigma, T L\left(\right.\right.$ Until, $\left.\left.\mathbf{K}^{-}\right)\right]$is interpreted as $\{a \in \mathcal{M} \mid \mathcal{M}, a \models A\}$. We say that first-order formulas in the signature $\mathcal{E}\left[\Sigma, T L\left(\right.\right.$ Until, $\left.\left.\mathbf{K}^{-}\right)\right] \cup\{<\}$ are equivalent over $\mathcal{M}$ (respectively, over a class of $\Sigma$-chains $\mathcal{C}$ ) if they are equivalent in the canonical $T L\left(U n\right.$ til, $\left.\mathbf{K}^{-}\right)$-expansion of $\mathcal{M}$ (in the canonical $T L\left(\right.$ Until, $\left.\mathbf{K}^{-}\right)$-expansion of every $\left.\mathcal{M} \in \mathcal{C}\right)$. The canonical $T L\left(\right.$ Since, $\left.\mathbf{K}^{+}\right)$expansion of a chain $\mathcal{M}$ is defined similarly.

The next lemma implies that Lemma 5.1 holds over the canonical $T L\left(\right.$ Since, $\left.\mathbf{K}^{+}\right)$-expansions and over canonical $T L\left(\right.$ Until, $\left.\mathbf{K}^{-}\right)$-expansions.

\section{Lemma 7.8.}

(1) $\neg\left[\alpha_{0}, \beta_{1} \ldots, \beta_{n-1}, \alpha_{n-1}, \beta_{n}, \alpha_{n}\right]\left(z_{0}, z_{1}\right)$ is equivalent over the canonical $T L\left(\right.$ Since, $\left.\boldsymbol{K}^{+}\right)$expansions of Dedekind complete chains to a $\left(z_{0}, z_{1}\right)-\vee \vec{\exists} \forall$-formula.

(2) Dually, $\neg\left[\alpha_{0}, \beta_{1} \ldots, \beta_{n-1}, \alpha_{n-1}, \beta_{n}, \alpha_{n}\right]\left(z_{0}, z_{1}\right)$ is equivalent over the canonical $T L\left(\right.$ Until, $\left.\boldsymbol{K}^{-}\right)$-expansions of Dedekind complete chains to a $\left(z_{0}, z_{1}\right)-\vee \vec{\exists} \forall$-formula.

Proof. Actually, our proof of Lemma 5.1, as it is, works for the canonical $T L\left(\right.$ Since, $\left.\mathbf{K}^{+}\right)$expansions of Dedekind complete chains, when " $\vec{\exists} \forall$ formulas" are replaced by "( $\left.z_{0}, z_{1}\right)-\vec{\exists} \forall$ formulas."

Indeed, Lemma 5.3 uses only modality $\mathbf{K}^{+}$. Thus, exactly the same proof works for $T L\left(\right.$ Since, $\mathbf{K}^{+}$)-expansions and for $T L\left(\mathbf{U n t i l}, \mathbf{K}^{-}\right.$)-expansions (because $\mathbf{K}^{+}$is equivalent to a $T L($ Until) formula).

In the proof of Corollary $5.4(1)$ we used Lemma 5.3 and Until modality. Hence, it holds for $T L\left(\right.$ Until, $\left.\mathbf{K}^{-}\right)$-expansions. Corollary 5.4(2) is dual and it holds for $T L\left(\right.$ Since, $\left.\mathbf{K}^{+}\right)$expansions.

In proof of Lemma 5.1 we use standard logical equivalences and Corollary 5.4(2). Hence, it works as it is for the canonical $T L\left(\right.$ Since, $\left.\mathbf{K}^{+}\right)$- expansions of Dedekind complete chains. This proves (1). Item (2) is the mirror image of (1).

Notation 7.9. We use the abbreviated notation $\left[\alpha_{0}, \beta_{1} \ldots, \alpha_{n-1}, \beta_{n}, \alpha_{n}, \beta_{n+1}\right]\left(z_{0}, \infty\right)$ for

$$
\begin{aligned}
\exists x_{n} \ldots \exists x_{1} \exists x_{0} z_{0}=x_{0} & \wedge\left(x_{n}>\cdots>x_{1}>x_{0}\right) \\
& \wedge \bigwedge_{j=0}^{n} \alpha_{j}\left(x_{j}\right) \wedge \bigwedge_{j=1}^{n}(\forall y)_{>x_{j-1}}^{<x_{j}} \beta_{j}(y) \wedge(\forall y)_{>x_{n}} \beta_{n+1}(y) ;
\end{aligned}
$$


such formulas will be called $\left(z_{0}, \infty\right)$-formulas; we use the similarly abbreviated notation $\left[\beta_{0}, \alpha_{0}, \beta_{1} \ldots, \alpha_{n-1}, \beta_{n}, \alpha_{n}\right]\left(-\infty, z_{0}\right)$ for the $\vec{\exists} \forall$-formula

$$
\begin{aligned}
\exists x_{n} \ldots \exists x_{1} \exists x_{0} z_{0}=x_{n} & \wedge\left(x_{n}>\cdots>x_{1}>x_{0}\right) \\
& \wedge \bigwedge_{j=0}^{n} \alpha_{j}\left(x_{j}\right) \wedge \bigwedge_{j=1}^{n}(\forall y)_{>x_{j-1}}^{<x_{j}} \beta_{j}(y) \wedge(\forall y)^{<x_{0}} \beta_{0}(y) .
\end{aligned}
$$

Lemma 7.10. $\left[\alpha_{0}, \beta_{1} \ldots, \alpha_{n-1}, \beta_{n}, \alpha_{n}, \beta_{n+1}\right]\left(z_{0}, \infty\right)$ over canonical TL(Until, $\left.\boldsymbol{K}^{-}\right)$-expansions is equivalent to a $T L\left(\right.$ Until, $\left.\boldsymbol{K}^{-}\right)$formula.

Proof. By a straightforward formalization as in the proof of Proposition 3.5.

Definition 7.11 (Syntactically future FOMLO formulas). A FOMLO formula $\varphi\left(z_{0}\right)$ is syntactically future if all its quantifiers are bounded quantifiers of the form $(\forall y)_{>z_{0}}$ and $(\exists y)_{>z_{0}}$.

The following lemma immediately follows from the definition.

Lemma 7.12. A syntactically future FOMLO formula is future. A FOMLO formula $\varphi\left(z_{0}\right)$ is future iff it is equivalent to a syntactically future FOMLO formula.

Definition 7.13. Let $\left(z_{0}, z_{1}, \ldots, z_{k}\right)$ be a sequence of distinct variables. A formula is $\left(z_{0}, z_{1}, \ldots, z_{k}, \infty\right)-\vec{\exists} \forall$ formula if it is a conjunction $\bigwedge_{i \leq k} \varphi_{i}$, where $\varphi_{k}$ is $\left(z_{k}, \infty\right)-\vec{\exists} \forall$ formula and $\varphi_{i}$ is $\left(z_{i}, z_{i+1}\right)-\vec{\exists} \forall$ formulas for $i<k$. A formula is a $\left(z_{0}, z_{1}, \ldots, z_{k}, \infty\right)-\vee \vec{\exists} \forall$ formula if it is equivalent to a disjunction of $\left(z_{0}, z_{1}, \ldots, z_{k}, \infty\right)-\vec{\exists} \forall$ formulas.

Lemma 7.14. Let $\varphi\left(z_{0}, z_{1}, \ldots, z_{k}\right)$ be a FOMLO formula with free variables in $\left\{z_{i} \mid i \leq k\right\}$ and all its quantifiers are bounded quantifiers of the form $(\forall y)_{>z_{0}}$ and $(\exists y)_{>z_{0}}$. Then, there is $\left(z_{0}, z_{1}, \ldots, z_{k}, \infty\right)-\vee \vec{\exists} \forall$ formula $\psi$ such that $z_{0}<z_{1}<\cdots<z_{k} \wedge \varphi$ is equivalent over the canonical TL(Until, $\left.\boldsymbol{K}^{-}\right)$-expansions of Dedekind complete chains to $z_{0}<z_{1}<\cdots<z_{k} \wedge \psi$.

Proof. By Lemmas 7.6, 7.8(2), 7.10 and a straightforward structural induction.

Now we are ready to prove Theorem 7.4 .

Proof. (of Theorem 7.4 Assume that $\varphi\left(z_{0}\right)$ is a future FOMLO formula. By Lemma 7.12 w.l.o.g we can assume that all its quantifiers are bounded quantifiers of the form $(\forall y)_{>z_{0}}$ and $(\exists y)_{>z_{0}}$. By 7.14, it is equivalent to $\left(z_{0}, \infty\right)-\vee \vec{\exists} \forall$ formula. Hence, by Lemma 7.10, it is equivalent to a $T L\left(\right.$ Until, $\left.\mathbf{K}^{-}\right)$formula. Therefore, by Lemma 7.3 it is equivalent to a syntactically future $T L\left(U_{n}\right.$ til, $\left.\mathbf{K}^{-}\right)$formula.

In 9] we erroneously stated that the analog of Proposition 4.3 holds for $T L\left(\right.$ Until, $\left.\mathbf{K}^{-}\right)$expansions. However, " $P$ is unbounded from below" is expressible by a FOMLO sentence $\forall x \exists y(y<x \wedge P(y))$; yet there is no $\vee \vec{\exists} \forall$ formula which expresses " $P$ is unbounded from below" over the canonical $T L\left(U n t i l, \mathbf{K}^{-}\right)$-expansions of integer-chains. We state the following Proposition for the sake of completeness.

Proposition 7.15. Every FOMLO formula is equivalent over the canonical TL(Until, $\left.\boldsymbol{K}^{-}\right)$expansions of Dedekind complete chains to a positive boolean combination of $\vec{\exists} \forall$ formulas and sentences of the form "P is unbounded from below." 
The additional step needed for the proof of Proposition 7.15 is an observation that $\neg\left[\beta_{0}, \alpha_{0}, \beta_{1} \ldots, \alpha_{n-1}, \beta_{n}, \alpha_{n}\right]\left(-\infty, z_{0}\right)$ is equivalent over the canonical $T L\left(U n t i l, \mathbf{K}^{-}\right)$-expansions of Dedekind complete chains to a positive boolean combinations of $\left(-\infty, z_{0}\right)-\vec{\exists} \forall$ formulas and sentences of the form " $P$ is unbounded from below." This is proved almost in the same way as Lemma 5.1 .

Acknowledgments I am very grateful to Yoram Hirshfeld for numerous insightful discussions, and to the anonymous referees for their helpful suggestions.

\section{REFERENCES}

[1] D. Gabbay. Expressive functional completeness in tense logic (preliminary report). In U. Monnich, editor, Aspects of Philosophical Logic, pages 91-117. Reidel, Dordrecht, 1981.

[2] D. Gabbay, I. Hodkinson, and M. Reynolds. Temporal logic: Mathematical Foundations and Computational Aspects. Oxford University Press, 1994.

[3] D. Gabbay, A. Pnueli, S. Shelah and J. Stavi. On the Temporal Analysis of Fairness. In POPL 1980, pp. 163-173, 1980.

[4] Y. Hirshfeld, A. Rabinovich. Future temporal logic needs infinitely many modalities. Information and Computation, 187(2003), 196-208.

[5] I. Hodkinson. Expressive completeness of Until and Since over dedekind complete linear time. Modal logic and process algebra, ed. A. Ponse, M. de Rijke, Y. Venema, CSLI Lecture Notes 53, 1995.

[6] I. Hodkinson. Notes on games in temporal logic. Lecture notes for LUATCS meting, Johannesburg, Dec 1999. http://www.doc.ic.ac.uk/ imh/index.html

[7] I. Hodkinson and M. Reynolds. Temporal Logic Chapter 11 (pp. 655-720) in Handbook of Modal Logic, Patrick Blackburn, Johan van Benthem, and Frank Wolter, eds., Elsevier Science, 2006.

[8] H. W. Kamp. Tense logic and the theory of linear order. Phd thesis, University of California, Los Angeles, 1968.

[9] D. Pardo and A. Rabinovich: A Finite Basis for 'Almost Future' Temporal Logic over the Reals. MFCS 2012: $740-751$

[10] A. Pnueli (1977). The temporal logic of programs. In Proc. IEEE 18th Annu. Symp. on Found. Comput. Sci., pages 46-57, New York, 1977.

This work is licensed under the Creative Commons Attribution-NoDerivs License. To view a copy of this license, visit http://creativecommons.org/licenses/by-nd/2.0/ or send a letter to Creative Commons, 171 Second St, Suite 300, San Francisco, CA 94105, USA, or Eisenacher Strasse 2, 10777 Berlin, Germany 